\title{
LEISHMANIOSE VISCERAL CANINA: BREVE REVISÃO E RELATOS DE CASOS
}

\author{
Vanessa Paulino da Cruz Vieira ${ }^{1}$ \\ Nathalia Mendes Figueiredo ${ }^{2}$
}

\begin{abstract}
RESUMO
A leishmaniose é uma doença causada por um protozoário da espécie Leishmania chagasi. É transmitida pela picada de fêmeas do inseto vetor infectado, um flebotomíneo da espécie Lutzomyia longipalpis, conhecido popularmente como mosquito palha. De ampla distribuição geográfica, o Brasil é responsável por $96 \%$ dos casos notificados nas Américas. Trata-se de uma zoonose de evolução crônica, com acometimento sistêmico e, se não tratada, pode levar a óbito até $90 \%$ dos casos. No cão, considerado o principal reservatório urbano do agente etiológico, a leishmaniose visceral canina é uma enfermidade que provoca grande sofrimento aos animais acometidos, tutores e contactantes. As manifestações clínicas dependem intrinsecamente do tipo de resposta imunológica apresentada pelo seu hospedeiro, podendo surgir de três meses até vários anos após a infecção e são, por vezes, inespecíficas. O diagnóstico laboratorial da leishmaniose visceral canina baseia-se na tríade de métodos sorológicos, parasitológicos e moleculares e é essencial para o estadiamento da doença e escolha do tratamento adequado. O tratamento possui como principais objetivos reduzir a carga parasitária do cão, neutralizando a sua capacidade infectante, restaurando adequadamente a sua resposta imune, promovendo a melhora clínica e prevenindo recaídas. $\mathrm{O}$ objetivo com esse trabalho foi apresentar uma revisão de literatura expositiva acerca dos aspectos epidemiológicos, diagnóstico, controle e manejo da leishmaniose visceral canina e apresentar relatos de casos de animais diagnosticados positivos atendidos em clínica veterinária particular em Belo Horizonte, Minas Gerais, do ponto de vista clínico e parasitológico, que apresentaram recidivas ao tratamento com a droga leishmanicida miltefosina. O acompanhamento dos casos clínicos apresentados possibilitou uma análise do emprego, aplicabilidade e eficiência dos métodos diagnósticos disponíveis para leishmaniose, e dos tratamentos atualmente disponíveis, sendo útil na orientação do médico veterinário na rotina clínica.
\end{abstract}

Palavras-chave: diagnóstico; tratamento; milteforan; estadiamento; Leishmania infantum.

\section{CANINE VISCERAL LEISHMANIOSIS: BRIEF REVIEW AND CASE REPORTS}

\begin{abstract}
Leishmaniasis is a disease caused by a protozoan of the species Leishmania chagasi. It is transmitted by the bite of females of the infected vector insect, a sand fly of the species Lutzomyia longipalpis, popularly known as the straw mosquito. With a wide geographical distribution, Brazil is responsible for $96 \%$ of the cases notified in the Americas. It is a chronic evolution zoonosis, with systemic involvement and, when untreated, can lead to death in up to $90 \%$ of cases. In dogs, considered the main urban reservoir of the etiologic agent, canine visceral leishmaniasis is a disease that causes great suffering to the affected animals, guardians and contacts. Clinical manifestations intrinsically depends on the type of immune response presented by its host, and can appear from three months to several years after

\footnotetext{
${ }^{1}$ Instituto Federal de Educação, Ciência e Tecnologia do Norte de Minas Gerais - Campus Salinas Correspondência vanessa.vieira@ifnmg.edu.br

${ }^{2}$ Universidade Federal de Viçosa, nathaliamfigueiredo@outlook.com
}

Vieira VPC, Figueiredo NM. Leishmaniose Visceral Canina: Breve revisão e relatos de casos. Vet. e Zootec. 2021; v28: 001-012. 
infection and are sometimes unspecific. The laboratory diagnosis of canine visceral leishmaniasis is based on the triad of serological, parasitological and molecular methods and is essential for the staging of the disease and choosing the appropriate treatment. The main objectives of the treatment are to reduce the parasitic burden of the dog, neutralizing its infectious capacity, properly restoring its immune response, promoting clinical improvement and preventing relapses. The objective of this work was to present a review of the literature on the epidemiological aspects, diagnosis, control and management of canine visceral leishmaniasis and to present case reports of positive diagnosed animals seen in a private veterinary clinic in Belo Horizonte, Minas Gerais, from the clinical and parasitological point of view, which presented relapses to treatment with the leishmanicidal drug miltefosine. The monitoring of the clinical cases presented made it possible to analyze the use, applicability and efficiency of the diagnostic methods available for leishmaniasis, and of the treatments currently available, being useful in the guidance of the veterinarian in the clinical routine.

Keywords: diagnosis; treatment; milteforan; staging; Leishmania infantum .

\section{LEISHMANIOSIS VISCERAL CANINA: BREVE REVISIÓN E INFORMES DE CASOS}

\section{RESUMEN}

La leishmaniasis es una enfermedad causada por un protozoo de la especie Leishmania chagasi. Se transmite por la picadura de las hembras del insecto vector infectado, una mosca de la especie Lutzomyia longipalpis, conocida popularmente como mosquito de la paja. Con una amplia distribución geográfica, Brasil es responsable del $96 \%$ de los casos notificados en las Américas. Es una zoonosis de evolución crónica, con afectación sistémica y, si no se trata, puede provocar la muerte hasta en $90 \%$ de los casos. En los perros, considerados los principales reservorios urbanos del agente etiológico, la leishmaniasis visceral canina es una enfermedad que causa un gran sufrimiento a los animales afectados, guardianes y contactos. Las manifestaciones clínicas dependen intrínsecamente del tipo de respuesta inmune que presente su hospedador, que pueden aparecer desde tres meses hasta varios años después de la infección y en ocasiones son inespecíficas. El diagnóstico de laboratorio de la leishmaniasis visceral canina se basa en la tríada de métodos serológicos, parasitológicos y moleculares y es fundamental para la estadificación de la enfermedad y la elección del tratamiento adecuado. Los principales objetivos del tratamiento son reducir la carga parasitaria del perro, neutralizando su capacidad infecciosa, restaurando adecuadamente su respuesta inmunitaria, favoreciendo la mejoría clínica y previniendo las recaídas. El objetivo de este trabajo fue presentar una revisión de la literatura sobre los aspectos epidemiológicos, diagnóstico, control y manejo de la leishmaniasis visceral canina y presentar informes de casos de animales diagnosticados positivos atendidos en una clínica veterinaria privada en Belo Horizonte, Minas Gerais, de el punto de vista clínico y parasitológico, que presentó recaídas al tratamiento con el fármaco leishmanicida miltefosina. El seguimiento de los casos clínicos presentados permitió analizar el uso, aplicabilidad y eficacia de los métodos diagnósticos disponibles para la leishmaniasis, y de los tratamientos actualmente disponibles, siendo de utilidad en la orientación del veterinario en la rutina clínica.

Palabras-clave: diagnóstico; tratamiento; milteforan; estadificación; Leishmania infantum. 


\section{INTRODUÇÃO}

A leishmaniose é uma doença causada por um protozoário da espécie Leishmania chagasi. É conhecida como calazar, esplenomegalia tropical e febre dundun. É transmitida pela picada de fêmeas do inseto vetor infectado, o flebotomíneo conhecido popularmente como mosquito palha. No Brasil, a principal espécie responsável pela transmissão é Lutzomyia longipalpis (1).

A leishmaniose cutânea (LC) e visceral (LV) é endêmica em 18 países das Américas, sendo que $96 \%$ dos casos de leishmaniose na região são reportados no Brasil, com um registro de 19.395 casos em 2015. A Organização Pan-Americana da Saúde - OPAS/OMS relata também um aumento de mortes causadas por LV desde 2012 e alcançando em 2016 uma taxa de letalidade de 7,9\% nas Américas, considerada a mais elevada quando comparada a outros continentes (2).

A LV segue apresentando uma ampla distribuição geográfica de casos humanos no Brasil, destacando-se as regiões Nordeste, Sudeste e Centro-Oeste. Trata-se de uma zoonose de evolução crônica, com acometimento sistêmico e, se não tratada, pode levar a óbito até $90 \%$ dos casos (3).

A leishmaniose possui ainda uma face social de países emergentes, ligada a problemas de sanidade, deficiências nutricionais e acesso à saúde pela população, além de acesso à educação. A pobreza está relacionada à ocorrência de leishmaniose, devido à aglomeração de pessoas em moradias, condições sanitárias inadequadas, criação de animais próximos às residências e mata no peridomicílio, que favorecem a dispersão do vetor e o seu contato com o ser humano (4-6).

No cão, considerado o principal reservatório urbano do agente etiológico, a leishmaniose visceral canina (LVC) é uma enfermidade que provoca grande sofrimento aos animais acometidos, tutores e contactantes; afeta a saúde física e o bem-estar da família, vizinhos e comunidade. O tratamento, além de não ser recomendado pelo Ministério da Saúde, possui um curso difícil e um custo muito elevado (7-9).

No cão, a doença se manifesta frequentemente com caquexia progressiva, hipergamaglobulinemia, hepatoesplenomegalia, anemia, linfadenopatia, úlceras de pele e focinho. Sem tratamento, possui uma evolução crônica e fatal $(8,10)$.

Embora a eutanásia de animais soropositivos seja adotada como uma das principais medidas de controle da doença pelo Ministério da Saúde e pelos Centros de Controle de Zoonoses há décadas, a prevalência da doença aumenta a cada ano, na população canina e humana, o que alerta para a necessidade de métodos diagnósticos mais precisos e de controle da disseminação mais eficientes (11-13).

O diagnóstico da LVC mostra-se complexo, uma vez que os sinais clínicos apresentados pelos animais são frequentemente inespecíficos e confundem-se com os sinais apresentados em outras doenças. O diagnóstico laboratorial da LVC baseia-se na tríade de métodos sorológicos, parasitológicos e moleculares $(14,15)$.

Além disso, é necessário ressaltar a diferença entre um animal doente e um animal soropositivo. A soropositividade de um animal diz respeito ao exame diagnóstico utilizado, e deve-se sempre levar em consideração a sensibilidade e especificidade de cada teste, além de avaliações quantitativas que diferenciam um animal infectado de um animal que foi exposto ao agente (16).

Os exames laboratoriais recomendados pelo Ministério da Saúde para avaliação da soroprevalência em inquéritos caninos amostrais e censitários são o imunoenzimático ELISA, utilizado para a triagem de cães e a imunofluorescência indireta - RIFI para a confirmação dos cães sororreagentes ao teste ELISA. Os exames sorológicos são empregados nos programas nacional e estadual de vigilância e controle da LVC e devem ser realizados nos 
laboratórios centrais estaduais (LACENs) ou como nos Unidades de Vigilância de Zoonoses (UVZs) municipais (3).

Quanto ao tratamento, são empregados agentes imunomoduladores, leishmanicidas e leishmanistáticos, de acordo com o estadiamento da doença no animal $(15,17,18)$.

Desde a regulamentação para o primeiro fármaco liberado pelo Ministério da Saúde para o tratamento da LVC, em 2016, muitos avanços têm sido feitos na terapêutica e no prognóstico da doença. De acordo com as Diretrizes do Brasileish, 2018 (19), o tratamento da leishmaniose deve ser instituído apenas após um diagnóstico preciso, e possui como principais objetivos reduzir a carga parasitária do cão, neutralizando a sua capacidade infectante, restaurando adequadamente a sua resposta imune, promovendo a melhora clínica e prevenindo recaídas.

Para isso, é altamente recomendado o estadiamento da doença, para auxiliar no manejo terapêutico do paciente de acordo com as suas necessidades e o estágio da doença em que se encontra, além de se estabelecer o seu prognóstico. O estadiamento requer um acurado exame clínico do animal, exames de imagem e exames de patologia clínica, que incluem hemograma, bioquímico e sorologia quantitativa para leishmaniose. Sollano-Gallego e colaboradores, propuseram em 2011 (16), um sistema de estadiamento, terapia e prognóstico da leishmaniose canina, que foi adaptado pelo Brasileish com drogas regulamentadas no Brasil.

O tratamento da LVC no Brasil utiliza protocolos variados que combinam o uso de drogas imunomoduladoras, leishmanicida e leishmaniostáticas (18).

As drogas imunomoduladoras podem ser empregadas como estimulantes ou supressoras. As drogas estimulantes incluem levamisol, domperidona, cimetidina e a própria vacina para leishmaniose em doses duplas (18). Uma das drogas mais empregadas na terapia imunomoduladora da LVC, a domperidona ativa a resposta imune celular por promover um aumento da concentração de prolactina sérica. Além de sua principal função como estimulante da produção de leite em mamíferos, a prolactina é classificada como uma citocina pró-inflamatória derivada de linfócitos, possuindo assim papel central na resposta imune $(20,21)$.

As drogas imunossupressoras são utilizadas no tratamento de distúrbios secundários à LVC provocados pela deposição de imunocomplexos. Essa deposição está associada ao aparecimento de poliartrite, vasculite e trombocitopenia imunomediados. Nesses casos, podese optar pelo uso de corticoides, como a prednisona e a dexametasona. Essa terapia diminui a fagocitose por macrófagos de plaquetas opsonizadas, possivelmente aumenta a produção de plaquetas e eventualmente diminui a produção de anticorpos (22).

Drogas leishmaniostáticas são aquelas que inibem a multiplicação do parasito. Empregada largamente nos protocolos terapêuticos de LVC em associação a outros medicamentos, o alopurinol é um leishmaniostático capaz de interromper a síntese proteica do parasito pela inibição do metabolismo das purinas, exercendo um efeito inibitório no crescimento da Leishmania. Possui efeitos colaterais incomuns e baixo custo. A principal utilidade dessa droga tem sido demonstrada no controle das recidivas da leishmaniose canina (23-25).

Autorizada pelo Ministério da Saúde em 2016, o Milteforan ${ }^{\mathrm{TM} 1}$, de princípio ativo miltefosina, é o único leishmanicida regulamentado para tratamento de LVC no Brasil. Embora o seu mecanismo de ação ainda não esteja completamente elucidado, é possível que este fármaco atue no parasito da mesma forma que atua em células tumorais, induzindo à apoptose e alterando as vias de sinalização celular mediada por lipídeos (26).

O tratamento da LVC é capaz de promover a melhora clínica do paciente, diminuição significativa da carga parasitária e menores chances de transferir L. infantum ao Lutzomyia longipalpis, demonstrado com PCR quantitativa antes e após o tratamento e xenodiagnóstico;

\footnotetext{
${ }^{1}$ Miltefosina. Virbac.
}

Vieira VPC, Figueiredo NM. Leishmaniose Visceral Canina: Breve revisão e relatos de casos. Vet. e Zootec. 2021; v28: 001-012. 
esses resultados atestam a validade do tratamento da LVC como medida de controle da doença $(27,28)$.

Assim, objetivou-se com esse trabalho, apresentar uma revisão de literatura expositiva acerca dos aspectos epidemiológicos, diagnóstico e manejo da LVC, bem como apresentar relatos de casos de cães diagnosticados positivos atendidos em clínica veterinária particular em Belo Horizonte, Minas Gerais, que foram refratários a protocolos de tratamento com a droga leishmanicida miltefosina.

\section{MATERIAIS E MÉTODOS}

Para a realização deste trabalho, foram utilizadas as recomendações de Preferred Reporting Items for Systematic Reviews and Meta-Analysis (PRISMA). Para isso, buscou-se os estudos distribuídos nos bancos de dados PubMed, além do Scielo (Scientific Electronic Library Online), Periódicos Capes e Science Direct, com as palavras-chave "Leishmania; diagnóstico; tratamento; milteforan estadiamento".

Para os relatos de casos, foram utilizados dados de registros de um Hospital Veterinário particular na região metropolitana de Belo Horizonte, Minas Gerais, Brasil, entre os anos 2017 e 2019.

Foram selecionados e avaliados os casos clínicos de nove cães portadores de LVC atendidos regularmente nesse hospital, que foram tratados com uso de miltefosina e apresentaram recidiva ao protocolo terapêutico. Para fins de apresentação, os casos foram numerados de 1 a 9 (QUADRO 1). Os casos foram avaliados quanto aos sinais clínicos dos pacientes, método diagnóstico empregado, protocolo terapêutico utilizado, terapia coadjuvante, tempo de tratamento.

Quadro 1. Análise de casos clínicos de cães diagnosticados positivos para leishmaniose visceral canina, atendidos em Hospital Veterinário particular em Belo Horizonte, Minas Gerais, Brasil, entre os anos 2017 e 2019.

\begin{tabular}{|c|c|c|c|c|c|}
\hline Caso & Raça & $\begin{array}{l}\text { Sinais } \\
\text { clínicos }\end{array}$ & $\begin{array}{c}\text { Método } \\
\text { diagnóstico }\end{array}$ & $\begin{array}{c}\text { Protocolo } \\
\text { terapêutico }\end{array}$ & $\begin{array}{c}\text { Terapia } \\
\text { coadjuvante }\end{array}$ \\
\hline 1 & Pinscher & $\begin{array}{l}\text { Nódulos nas } \\
\text { mamas e } \\
\text { próximo aos } \\
\text { olhos }\end{array}$ & $\begin{array}{l}\text { Histopatológico; } \\
\text { ELISA e RIFI }\end{array}$ & $\begin{array}{l}\text { Mitelfosina a cada } 24 \\
\text { horas, por } 28 \text { dias; } \\
\text { alopurinol a cada } 12 \\
\text { horas, recomendação } \\
\text { de uso contínuo. }\end{array}$ & Mastectomia parcial \\
\hline 2 & Poodle & $\begin{array}{l}\text { Sangramento } \\
\text { nasal intenso, } \\
\text { prurido e } \\
\text { otite bilateral }\end{array}$ & $\begin{array}{l}\text { Teste rápido de } \\
\text { leishmaniose com Kit } \\
\text { Alere }{ }^{1}{ }^{1} \text {, com } \\
\text { resultado não } \\
\text { reagente; ELISA e } \\
\text { RIFI reagentes. } \\
\end{array}$ & $\begin{array}{c}\text { Alopurinol e } \\
\text { domperidona, a cada } 12 \\
\text { horas, com uso } \\
\text { contínuo; miltefosina a } \\
\text { cada } 24 \text { horas, por um } \\
\text { período de } 28 \text { dias } \\
\end{array}$ & $\begin{array}{l}\text { Duas doses de ácido } \\
\text { tranexâmico por via } \\
\text { oral, com intervalo de } \\
12 \text { horas, para controle } \\
\text { do sangramento nasal. }\end{array}$ \\
\hline 3 & Pug & $\begin{array}{l}\text { Ceratoconjun } \\
\text { tivite seca }\end{array}$ & $\begin{array}{c}\text { teste rápido para } \\
\text { leishmaniose com Kit } \\
\text { Imunocromatografia; } \\
\text { ELISA e RIFI; } \\
\text { punção de medula } \\
\text { óssea, com realização } \\
\text { de exame citológico e } \\
\text { mielograma }\end{array}$ & $\begin{array}{c}\text { Alopurinol e } \\
\text { domperidona, a cada } 12 \\
\text { horas, com uso } \\
\text { contínuo; } \\
\text { miltefosina a cada } 24 \\
\text { horas, por um período } \\
\text { de } 28 \text { dias }\end{array}$ & $\begin{array}{c}\text { Colírio a base de } \\
\text { trometamol cetrolaco, } \\
\text { com aplicação de uma } \\
\text { gota a cada } 6 \text { horas, até } \\
\text { desaparecimentos dos } \\
\text { sinais clínicos }\end{array}$ \\
\hline
\end{tabular}

Continua...

Vieira VPC, Figueiredo NM. Leishmaniose Visceral Canina: Breve revisão e relatos de casos. Vet. e Zootec. 2021; v28: 001-012. 
Contínuação do Quadro1.

\begin{tabular}{|c|c|c|c|c|c|}
\hline Caso & Raça & $\begin{array}{l}\text { Sinais } \\
\text { clínicos }\end{array}$ & $\begin{array}{c}\text { Método } \\
\text { diagnóstico }\end{array}$ & $\begin{array}{c}\text { Protocolo } \\
\text { terapêutico }\end{array}$ & $\begin{array}{c}\text { Terapia } \\
\text { coadjuvante }\end{array}$ \\
\hline 4 & SRD & Ausentes & $\begin{array}{c}\text { ELISA e RIFI; } \\
\text { citologia com } \\
\text { punção de medula } \\
\text { óssea }\end{array}$ & $\begin{array}{c}\text { Alopurinol e } \\
\text { domperidona, a cada } 12 \\
\text { horas, com uso } \\
\text { contínuo; } \\
\text { miltefosina a cada } 24 \\
\text { horas, por um período } \\
\text { de } 28 \text { dias }\end{array}$ & Ausente \\
\hline 5 & SRD & $\begin{array}{c}\text { Dor, } \\
\text { claudicação e } \\
\text { diarreia com } \\
\text { sangue }\end{array}$ & $\begin{array}{l}\text { ELISA reagente; } \\
\text { RIFI não reagente; } \\
\text { pesquisa de } \\
\text { hemoparasitas com } \\
\text { sangue periférico: } \\
\text { detectada co- } \\
\text { infecção de } \\
\text { Leishmania sp. e } \\
\text { Erlichia } \mathrm{sp} \\
\end{array}$ & $\begin{array}{c}\text { Alopurinol e } \\
\text { domperidona, a cada } 12 \\
\text { horas, com uso } \\
\text { contínuo; } \\
\text { miltefosina a cada } 24 \\
\text { horas, por um período } \\
\text { de } 28 \text { dias }\end{array}$ & $\begin{array}{l}\text { Predinisona a cada } 24 \\
\text { horas, tramadol e } \\
\text { dipirona a cada } 8 \text { horas, } \\
\text { durante } 3 \text { dias. } \\
\text { Doxiciclina a cada } 12 \\
\text { horas, por } 28 \text { dias. }\end{array}$ \\
\hline 6 & Pug & $\begin{array}{l}\text { Ausentes; } \\
\text { Durante } \\
\text { tratamento, } \\
\text { apresentou } \\
\text { episódios de } \\
\text { diarreia }\end{array}$ & ELISA e RIFI & $\begin{array}{c}\text { Alopurinol e } \\
\text { domperidona, a cada } 12 \\
\text { horas, com uso } \\
\text { contínuo; } \\
\text { miltefosina a cada } 24 \\
\text { horas, por um período } \\
\text { de } 28 \text { dias }\end{array}$ & $\begin{array}{l}\text { Metronidazol a cada } \\
\text { doze horas durante } \\
\text { cinco dias. }\end{array}$ \\
\hline 7 & SRD & Ausente & $\begin{array}{l}\text { ELISA e RIFI; } \\
\text { citologia com } \\
\text { punção de medula } \\
\text { óssea }\end{array}$ & $\begin{array}{c}\text { Alopurinol e } \\
\text { domperidona, a cada } 12 \\
\text { horas, com uso } \\
\text { contínuo; } \\
\text { miltefosina a cada } 24 \\
\text { horas, por um período } \\
\text { de } 28 \text { dias } \\
\end{array}$ & Ausente \\
\hline 8 & SRD & $\begin{array}{c}\text { Dermatite e } \\
\text { prurido }\end{array}$ & $\begin{array}{c}\text { Teste rápido para } \\
\text { leishmaniose com } \\
\text { Kit } \\
\text { Imunocromatografi } \\
\text { a; ELISA e RIFI. }\end{array}$ & $\begin{array}{c}\text { Alopurinol e } \\
\text { domperidona, a cada } 12 \\
\text { horas, com uso } \\
\text { contínuo; } \\
\text { miltefosina a cada } 24 \\
\text { horas, por um período } \\
\text { de } 28 \text { dias }\end{array}$ & $\begin{array}{l}\text { Predinisona, a cada } 24 \\
\text { horas, por via oral, } \\
\text { durante } 7 \text { dias }\end{array}$ \\
\hline 9 & Maltês & $\begin{array}{l}\text { Nódulos ao } \\
\text { redor dos } \\
\text { olhos }\end{array}$ & $\begin{array}{l}\text { Teste rápido para } \\
\text { leishmaniose com } \\
\text { Kit } \\
\text { Imunocromatografi } \\
\text { a; RIFI; citologia } \\
\text { com punção de } \\
\text { medula óssea }\end{array}$ & $\begin{array}{c}\text { Alopurinol e } \\
\text { domperidona, a cada } 12 \\
\text { horas, com uso } \\
\text { contínuo; } \\
\text { miltefosina a cada } 24 \\
\text { horas, por um período } \\
\text { de } 28 \text { dias }\end{array}$ & Ausente \\
\hline
\end{tabular}

${ }^{1}$ Allere; Leishmaniose Ac Test Kit. Detecção por Imunocromatografia dos anticorpos da Leishmaniose Visceral Canina.

A recidiva da doença foi constatada em retorno, recomendado aos pacientes após três meses do término do ciclo de tratamento da mitelfosina. Novo ciclo de tratamento foi instituído.

\section{DISCUSSÃO}

Com relação à sintomatologia clínica e diagnóstico positivo para LVC, não se observou no presente estudo de casos nenhuma associação entre maiores títulos de anticorpos ou maior

Vieira VPC, Figueiredo NM. Leishmaniose Visceral Canina: Breve revisão e relatos de casos. Vet. e Zootec. 2021; v28: 001-012. 
parasitismo detectado e presença de sinais clínicos característicos da leishmaniose, o que reafirma a importância do cão assintomático e oligossintomático na epidemiologia da leishmaniose, em concordância com o atestado por Dantas-Torres, Brito e Brandão-Filho (29) e Vasconsellos (30).

Quanto aos métodos diagnósticos, os testes ELISA e RIFI com diluição total foram os mais empregados nos casos de estudo, para triagem e estadiamento. Conforme descrito por Solano-Gallego et al. (16), esses testes são particularmente eficientes por fornecerem uma análise quantitativa por titulação dos anticorpos, o que é essencial para o correto estadiamento e escolha do tratamento adequado da leishmaniose.

O caso 5 apresentou discordância entre os exames RIFI e ELISA, em que o teste ELISA foi positivo e o teste RIFI não reagente, com uma titulação inferior a 40, configurando um diagnóstico indeterminado. A discordância entre as duas técnicas pode se dever à maior capacidade de detecção de anticorpos do exame ELISA em relação à RIFI. Mancianti et al. (31) demonstram, em um estudo comparativo entra as técnicas ELISA e RIFI no diagnóstico da leishmaniose canina, que o exame ELISA demonstra ser mais sensível que a RIFI (99,5\% e $98,4 \%$ ), enquanto possui uma menor especificidade (97,1\% e $100 \%)$, ainda que não sejam significativamente diferentes.

Além disso, conforme descrito por Laurenti (17) é grande o número de reações cruzadas dos testes sorológicos com anticorpos de outras tripanossomíases, levando a resultados falsopositivos. Corroborando essa informação, outros autores relatam que a ocorrência de coinfecções em hemoparasitoses, especialmente erliquiose e leishmaniose, foi descrita em diversos trabalhos e pode frequentemente ser confundida com uma reação cruzada em exames sorológicos $(32,33)$.

Para a confirmação do diagnóstico em casos indeterminados, são utilizados os exames parasitológicos, citologia e imunohistoquímica, que consistem na visualização direta da Leishmania, e moleculares, especialmente a Reação em Cadeia da Polimerase (PCR). Esses exames foram amplamente empregadas no diagnóstico definitivo dos casos de estudo por se tratarem de técnicas definitivas, seguras e 100\% específicas, cuja sensibilidade varia de acordo com o local da amostra (34). No caso 5, foi utilizada citologia de amostra de sangue periférico, que permitiu a visualização de formas amastigotas de Leishmania sp. e presença de Erlichia sp., caracterizando uma co-infecção.

Quanto ao protocolo de estadiamento e tratamento da leishmaniose, os casos relatados se encontram em concordância com as diretrizes dos grupos de pesquisa Brasileish e Leishvet $(16,19)$.

Todos os casos do presente estudo sofreram recidivas da infecção após o tratamento com miltefosina, detectadas no acompanhamento periódico realizado pelos animais, que tiveram que ser submetidos a novo ciclo de tratamento. Em cães, a leishmaniose apresenta maior resistência do que em humanos e as recidivas são frequentes, geralmente ocorrendo de seis meses a dois anos após o término do tratamento; essa resistência, embora ainda não esteja totalmente elucidada, pode ser atribuída tanto ao alojamento do parasito em certos tecidos quanto à resistência desenvolvida aos fármacos utilizados $(35,36)$.

Foi possível observar, durante o acompanhamento dos casos clínicos, grande comprometimento dos tutores em seguir adequadamente o tratamento instituído, realização dos exames solicitados e comparecimento aos retornos periódicos. O médico veterinário oncologista, responsável pelos casos de leishmaniose no Hospital Veterinário em estudo, atribui esse comprometimento a uma construção, baseada no diálogo e conscientização do médico veterinário com o tutor.

Vieira VPC, Figueiredo NM. Leishmaniose Visceral Canina: Breve revisão e relatos de casos. Vet. e Zootec. 2021; v28: 001-012. 


\section{CONSIDERAÇÕES FINAIS}

A leishmaniose visceral canina é uma doença de ampla distribuição no território brasileiro, que compromete a saúde e qualidade de vida dos cães acometidos, além de representar um grande desafio quanto às medidas de controle e prevenção adotadas.

O acompanhamento dos casos clínicos apresentados possibilitou uma análise do emprego, aplicabilidade e eficiência dos métodos diagnósticos disponíveis para leishmaniose, sendo útil na orientação do médico veterinário na rotina clínica.

Foi demonstrado que precisão diagnóstica é essencial para se optar pela melhor conduta veterinária possível, avaliação do prognóstico e possibilidade de tratamento da LVC, além de se evitar eutanásia de animais sadios diagnosticados erroneamente.

Além disso, as recidivas detectadas demonstram a importância e necessidade de acompanhamento periódico vitalício durante e após o tratamento. Apontam também para o uso racional e adequado desses fármacos, para evitar resistência e para a necessidade de novos protocolos de tratamento.

Por fim, ressalta-se a necessidade de medidas de controle da LVC que sejam voltadas para a Saúde Única, e não apenas para a eliminação do cão soropositivo, com o trabalho integrado e colaboração entre o governo municipal, agentes de saúde, tutores de cães, médicos veterinários e toda a população.

\section{REFERÊNCIAS}

1. Brasil. Ministério da Saúde. Leishmaniose visceral: o que é, causas, sintomas, tratamento, diagnóstico e prevenção [Internet]. Brasília: Ministério da Saúde; 2019 [citado 13 Nov 2019]. Disponível em: http://saude.gov.br/saude-de-a-z/leishmaniose-visceral

2. Pan American Health Organization, World Health Organization. Plan of action to strengthen surveillance and control of leishmaniasis in the americas 2017 - 2022 [Internet]. Washington: PAHO, WHO; 2017 [citado 5 Dez 2019]. Disponível em: http://iris.paho.org/xmlui/bitstream/handle/123456789/34147/PlanactionLeish20172022eng.pdf? sequence $=5 \&$ isAllowed $=y$

3. Brasil. Ministério da Saúde. Secretaria de Vigilância em Saúde. Departamento de Vigilância Epidemiológica. Manual de vigilância e controle da leishmaniose visceral [Internet]. Brasília: Ministério da Saúde; 2006 [citado 7 Fev 2021]. Disponível em: http://bvsms.saude.gov.br/bvs/publicacoes/manual_vigilancia_controle_leishmaniose_visc eral.pdf

4. World Health Organization. Research priorities for chagas disease, human african trypanosomiasis and leishmaniasis [Internet]. Genebra: WHO; 2012 [citado 7 Fev 2021]. (Technical report series, $\mathrm{n}^{\mathrm{o}}$ 975). Disponível em: https://apps.who.int/iris/handle/10665/77472

5. Dantas-Torres F. The role of dogs as reservoirs of Leishmania parasites, with emphasis on Leishmania (Leishmania) infantum and Leishmania (Viannia) braziliensis. Vet Parasitol [Internet]. 2007 [citado 7 Fev 2021];149(3-4):139-46. Disponível em: https://www.sciencedirect.com/science/article/abs/pii/S0304401707003597 
6. Machado CJS, Silva EG, Vilani RM. O uso de um instrumento de política de saúde pública controverso: a eutanásia de cães contaminados por leishmaniose no Brasil. Saude Soc. 2016;25(1):247-58. doi: https://doi.org/10.1590/S0104-12902016146918.

7. Lima CC, Grisotti M. Relação humano-animal e leishmaniose: repercussões no cotidiano de indivíduos inseridos em região endêmica. Saude Soc [Internet]. 2018 [citado 7 Fev 2021];27(4):1261-9. Disponível em: https://www.scielo.br/scielo.php?pid=S0104$12902018000401261 \&$ script $=$ sci_abstract\&tlng=pt

8. Brasil. Ministério da Saúde. Secretaria de Vigilância em Saúde. Coordenação-Geral de Desenvolvimento da Epidemiologia em Serviços. Guia de vigilância em saúde : [Internet]. Brasília: Ministério da Saúde; 2017 [citado 7 Fev 2021]. v. 3. Disponível em: https://antigo.saude.gov.br/images/pdf/2017/setembro/05/Guia-de-Vigilancia-em-Saude2017-Volume-3.pdf

9. Castanheira ARF. A farmacoeconomia aplicada à medicina veterinária: análise de custos comparada entre o tratamento e a vacinação da leishmaniose canina [dissertação] [Internet]. Lisboa: Universidade de Lisboa; 2013 [citado 7 Fev 2021]. Disponível em: http://biblioteca.phorteeducacional.com.br/items/1152830

10. Silva FS. Patologia e patogênese da leishmaniose visceral canina. Rev Trop Cienc Agrar Biol [Internet]. 2007 [citado 7 Fev 2021];1(1):20. Disponível em: https://www.researchgate.net/profile/Francinaldo-

Silva/publication/220000420 Patologia_e patogenese da leishmaniose visceral_canina/1 inks/54610d070cf2c1a63bff $7 \mathrm{bdb} /$ Patologia-e-patogenese-da-leishmaniose-visceralcanina.pdf

11. Zuben APB, Donalísio MR. Dificuldades na execução das diretrizes do Programa de Vigilância e Controle da Leishmaniose Visceral em grandes municípios brasileiros. Cad Saude Publica. 2016;32(6):20. doi: https://doi.org/10.1590/0102-311X00087415.

12. Silva JD, Melo DHM, Costa JAG, Costa DF, Silva RBS, Melo MA, et al. Leishmaniose visceral em cães de assentamentos rurais. Pesqui Vet Bras [Internet]. 2017 [citado 7 Fev 2021];37(11):1292-8. Disponível em: https://www.scielo.br/pdf/pvb/v37n11/1678-5150pvb-37-11-01292.pdf

13. Andre LPP, Fonseca ZAA, Ribeiro WLC. Análise dos casos de leishmaniose humana e sua relação com a eutanásia de animais recolhidos pelo centro de controle de zoonoses de Mossoró RN. Rev Bras Hig Sanidade Anim [Internet]. 2013 [citado 7 Fev 2021];7(2):21224. Disponível em: https://dialnet.unirioja.es/servlet/articulo?codigo=5203823

14. Laurenti MD. Correlação entre o diagnóstico parasitológico e sorológico na leishmaniose visceral americana canina. BEPA Bol Epidemiol Paul [Internet]. 2009 [citado 7 Fev 2021];6(67):13-23. Disponível em: http://periodicos.ses.sp.bvs.br/scielo.php?script=sci_arttext\&pid=S1806$42722009000700002 \& \operatorname{lng}=$ pt\&nrm=iso

15. Faria AR, Andrade HM. Diagnóstico da Leishmaniose Visceral Canina: grandes avanços tecnológicos e baixa aplicação prática. Rev Pan-Amazonica Saude [Internet]. 2012 [citado 7

Fev 2021];3(2):47-57.

Disponível

em:

Vieira VPC, Figueiredo NM. Leishmaniose Visceral Canina: Breve revisão e relatos de casos. Vet. e Zootec. 2021; v28: 001-012. 
http://scielo.iec.gov.br/scielo.php?script=sci_abstract\&pid=S2176$62232012000200007 \& \operatorname{lng}=$ pt\&nrm=iso

16. Solano-Gallego L, Miró G, Koutinas A, Cardoso L, Pennisi MG, Ferrer L, et al. LeishVet guidelines for the practical management of canine leishmaniosis. Parasit Vectors. 2011;4:86. doi: 10.1186/1756-3305-4-86.

17. Laurenti MD. Patologia e patogenia das leishmanioses [tese] [Internet]. São Paulo: Universidade de São Paulo; 2010 [citado 7 Fev 2021]. Disponível em: https://www.teses.usp.br/teses/disponiveis/livredocencia/10/tde-26112010105228/publico/Tese_LD_Marcia_Dalastra_Laurenti_2010_A.pdf

18. Tabanez P, Ribeiro V. Estadiamento e tratamento da LVC [Internet]. Belo Horizonte: Brasileish; 2017 [citado 13 Nov 2019]. Disponível em: https://www.brasileish.com.br/assets/files/brasileish18_12_2017.pdf

19. Brasileish. Diretrizes para o diagnóstico, estadiamento, tratamento e prevenção da leishmaniose canina. Diretrizes [Internet]. Belo Horizonte: Brasileish; 2018 [citado 13 Nov 2019]. Disponível em: https://www.brasileish.com.br/diretrises.html

20. Hinterberger-Fischer M. Prolactin as pro-inflammatory cytokine - considerations on consolidated immunotherapy after high dosage therapy. Acta Med Austriaca Suppl [Internet]. 2000 [citado 7 Fev 2021];52:16-20. Disponível em: https://pubmed.ncbi.nlm.nih.gov/11261272/

21. Passos SR. Tratamento clínico da leishmaniose tegumentar americana canina com furazolidona e domperidona [dissertação] [Internet]. Alegre (ES): Universidade Federal do Espírito Santo; 2014 [citado 7 Fev 2021]. Disponível em: https://repositorio.ufes.br/handle/10/7781

22. Brites MG. Trombocitopenia imunomediada em cães: revisão bibliográfica e relato de casos [monografia] [Internet]. Porto Alegre: Universidade Federal do Rio Grande do Sul; 2007 [citado 7 Fev 2021]. Disponível em: https://www.lume.ufrgs.br/handle/10183/13361

23. Ginel PJ, Lucena R, López R, Molleda JM. Use of allopurinol for maintenance of remission in dogs with leismaniasis. J Small Anim Pract [Internet]. 1998 [citado 7 Fev 2021];39(6):271-4. Disponível em: https://pubmed.ncbi.nlm.nih.gov/9673902/

24. Oliveira AC, Antonio NA, Piccinin A. Controle e tratamento da leishmaniose visceral canina. Rev Cient Eletronica Med Vet [Internet]. 2008 [citado 7 Fev 2021];VI(10):1-5. Disponível em: http://faef.revista.inf.br/imagens_arquivos/arquivos_destaque/filaqiOy9mWp4ki_2013-529-10-36-13.pdf

25. Fonseca Júnior JD. Casuísticas de cães atendidos com leishmaniose no hospital veterinário do centro universitário luterano de palmas durante o período de agosto a dezembro de 2018 [trabalho de conclusão de curso] [Internet]. Palmas: Centro Universitário Luterano de Palmas; 2019 [citado 7 Fev 2021]. Disponível em: encurtador.com.br/acpuI 
26. Soares-Bezerra R, Leon L, Genestra M, Braz J. Recentes avanços da quimioterapia das leishmanioses: moléculas intracelulares como alvo de fármacos. RBCF Rev Bras Cienc Farm [Internet]. 2004 [citado 7 Fev 2021];40(2):139-49. Disponível em: https://www.scielo.br/scielo.php?pid=S1516-

93322004000200003\&script=sci_abstract\&tlng=pt

27. Nery G, Becerra DRD, Borja LS , Magalhães-Junior JT, Souza BMPS, Franke CR, et al. Avaliação da infectividade parasitária a Lutzomyia longipalpis por xenodiagnóstico em cães tratados para leishmaniose visceral naturalmente adquirida. Pesqui Vet Bras. 2017;37(7):701-7. doi: 10.1590/S0100-736X2017000700009.

28. Vides JP, Moraes LRS. Tratamento da Leishmaniose visceral canina com miltefosina relatos de casos. Rev Educ Contin Med Vet Zootec CRMV-SP [Internet]. 2018 [citado 7 Fev 2021];16(3):80. Disponível em: https://www.revistamvezcrmvsp.com.br/index.php/recmvz/article/view/37830

29. Dantas-Torres F, Brito ME, Brandão-Filho SP. Seroepidemiological survey on canine leishmaniasis among dogs from an urban area of Brazil. Vet Parasitol [Internet]. 2006 [citado 7 Fev 2021];140(1-2):54-60. Disponível em: https://pubmed.ncbi.nlm.nih.gov/16621286/

30. Marcondes M, Vasconcellos SA. Leishmaniose Visceral [Internet]. São Paulo: CRMVSP; 2019 [citado 13 Nov 2019]. (Leishamniose - Serie Zoonoses). Disponível em: https://www.crmvsp.gov.br/arquivo_zoonoses/LEISHMANIOSE_SERIE_ZOONOSES.p df

31. Mancianti F, Pedonese F, Poli A. Evaluation of dot enzyme-linked immunosorbent assay (dot-ELISA) for the serodiagnosis of canine leishmaniosis as compared with indirect immunofluorescence assay. Vet Parasitol [Internet]. 1996 [citado 7 Fev 2021];65(1-2):1-9. Disponível em: https:/www.sciencedirect.com/science/article/abs/pii/0304401796009466

32. Diniz PPV. Miocardite em cães com erliquiose monocítica [tese] [Internet]. Botucatu: Universidade Estaudal Paulista; 2006 [citado 7 Fev 2021]. Disponível em: https://bv.fapesp.br/pt/dissertacoes-teses/4023/miocardite-em-caes-com-erliquiosemonocitica

33. Sousa KCM. Co-infecção por Ehrlichia canis, Leishmania chagasi e Babesia canis em cães naturalmente infectados em campo grande, mato grosso do sul [dissertação] [Internet]. Jaboticabal: Faculdade de Ciências Agrárias e Veterinárias, Universidade Estadual Paulista; 2012 [citado 13 Nov 2019]. Disponível em: https://repositorio.unesp.br/bitstream/handle/11449/96002/sousa_kcm_me_jabo.pdf?seque nce $=1$

34. Gontijo CM, Melo MN. Leishmaniose visceral no Brasil: quadro atual, desafios e perspectivas. Rev Bras Epidemiol. 2004;7(3):338-49. doi: https://doi.org/10.1590/S1415790X2004000300011.

35. Schimming BC. Leishmaniose visceral canina: revisão de literatura. Rev Cient Eletronica Med Vet [Internet]. 2012 [citado 7 Fev 2021];X(19):1-17. Disponível em: 
http://faef.revista.inf.br/imagens_arquivos/arquivos_destaque/QKOIwlDa047cxSZ_20136-24-15-1-25.pdf

36. Moreno AM, Nieto LCG, Rodríguez SH, Corrales GM, Campillo MC, Bautista MG, et al. Parasitosis sistémicas. In: Campillo MC, Vazquez FAR, Fernandez ARM, Acedo MCS, Rodriguez SH, Lopez-Cozar. IN et al. Parasitología veterinária. 1a ed. Madrid: McGrawHill Interamericana; 1999. p.651-665.

Recebido em: 19/03/2021

Aceito em: 17/06/2021 